\title{
Local retention, dispersal and fluctuating connectivity among populations of a coral reef fish
}

\author{
J. Derek Hogan · Roger J. Thiessen • \\ Peter F. Sale $\cdot$ Daniel D. Heath
}

Received: 12 April 2010/Accepted: 16 June 2011/Published online: 7 July 2011

(C) Springer-Verlag 2011

\begin{abstract}
The persistence and resilience of marine populations in the face of disturbances is directly affected by connectivity among populations. Thus, understanding the magnitude and pattern of connections among populations and the temporal variation in these patterns is critical for the effective management and conservation of marine species. Despite recent advances in our understanding of marine connectivity, few empirical studies have directly measured the magnitude or pattern of connections among populations of marine fishes, and none have explicitly investigated temporal variation in demographic connectivity. We use genetic assignment tests to track the dispersal of 456 individual larval fishes to quantify the extent of connectivity, dispersal, self-recruitment and local retention within and among seven populations of a coral reef fish (Stegastes partitus) over a three-year period. We found that some larvae do disperse long distances ( $\sim 200 \mathrm{~km})$; however, self-recruitment was a regular
\end{abstract}

Communicated by Craig Osenberg.

Electronic supplementary material The online version of this article (doi:10.1007/s00442-011-2058-1) contains supplementary material, which is available to authorized users.

J. D. Hogan · R. J. Thiessen · D. D. Heath

Great Lakes Institute for Environmental Research, University of Windsor, 401 Sunset Ave., Windsor, ON N9C 3P4, Canada

P. F. Sale

Institute for Water, Environment and Health, United Nations

University, Hamilton, ON L8P 0A1, Canada

Present Address:

J. D. Hogan $(\bowtie)$

Center for Limnology, University of Wisconsin-Madison, 680 North Park St., Madison, WI 53706, USA

e-mail: jdhogan2@wisc.edu phenomenon. Importantly, we found that dispersal distances, self-recruitment, local retention and the pattern of connectivity varied significantly among years. Our data highlight the unpredictable nature of connectivity, and underscore the need for more, temporally replicated, empirical measures of connectivity to inform management decisions.

Keywords Local retention - Self-recruitment - Dispersal kernel $\cdot$ Connectivity matrix $\cdot$ Metapopulation $\cdot$ Marine protected areas

\section{Introduction}

Coastal marine fisheries and ecosystems are in global decline due to the increasing intensity and diversity of stresses in coastal waters (Hutchings 2000; Jackson et al. 2001). Marine protected areas (MPAs) have been advocated as one measure to facilitate continued ecosystem viability (Sale et al. 2005). However, the efficacy of MPAs as well as the persistence and resilience of marine populations in the face of disturbances in general are fundamentally linked to the scale of dispersal and the degree of connectivity among populations (Eckert 2003; Botsford et al. 2009). In addition, dispersal and connectivity drive population genetic differentiation and thus play key roles in the evolution of local adaptation (Bradbury et al. 2008; Walter et al. 2009).

The degree of temporal stability in larval dispersal connecting marine populations has not been empirically tested, yet temporal variability in dispersal and connectivity will determine predictability in population dynamics and will impact population genetic structure (Larson and Julian 1999; Selkoe et al. 2006; Hogan et al. 2010). In fact, 
temporal changes in larval migration patterns have been implicated as a source of spatial and temporal patchiness in the genetic structure of marine populations (Selkoe et al. 2006; Hogan et al. 2010). Therefore, not only must we improve our understanding of larval dispersal pathways, local recruitment rates and population connectivity, we also need empirical estimates of the temporal component of variation in those variables for the effective management and conservation of marine populations (Sale et al. 2005; Levin 2006; Jones et al. 2007). It is logistically difficult to track the dispersal of small pelagic larvae in the marine environment, although technologies have improved in recent years such that connectivity studies are more feasible (Thorrold et al. 2002). Despite recent advances in the field of marine connectivity, relatively few empirical studies have measured the magnitude or pattern of contemporary connections among populations of marine fishes (Sale et al. 2005; Jones et al. 2009). In fact, most studies only estimate self-recruitment, the ratio of locally produced settlement to settlement from elsewhere (Jones et al. 1999, 2005; Swearer et al. 1999; Almany et al. 2007; Christie et al. 2010). Although self-recruitment is important, in order to assess population persistence we need estimates of local retention, the ratio of locally produced settlement to the total number of settlers locally produced, not selfrecruitment (Botsford et al. 2009). This metric is much harder to estimate as it requires information about larval exchange among a network of populations. Few studies have tracked larval dispersal among populations (Miller and Shanks 2004; Becker et al. 2007; Planes et al. 2009; Shima and Swearer 2009); fewer still have empirically estimated temporal variation in dispersal (Miller and Shanks 2004); and none have empirically investigated the degree of temporal stability in connectivity among a network of populations.

Coral reef fishes live in spatially patchy reef environments where populations are connected primarily by pelagic larval dispersal via ocean currents (Sale 1980). The duration of the pelagic larval stage in coral reef fishes is typically weeks or months (Leis 1991), providing the opportunity for long-distance dispersal. Biophysical models have estimated that the spatial scale of connectivity among reefs in the Caribbean is on the order of tens to hundreds of kilometers (Cowen et al. 2006). Recent empirical studies have shown that reef fish larvae have the ability, and perhaps the propensity, to return to their natal reef following a residency in the pelagic environment (Jones et al. 2005; Almany et al. 2007); they also may act to minimize long-distance dispersal (Fisher and Bellwood 2003). Thus, local processes have the potential to contribute substantially to the demography of reef fish populations.
Several recent population-genetic studies of a common Caribbean coral reef fish, Stegastes partitus (bicolor damselfish), shed some light on the spatial and temporal scales of gene flow (i.e., historical connectivity) among populations. Only one of these studies investigates contemporary connectivity (Christie et al. 2010). Purcell et al. (2009) found weak population structuring across the Caribbean basin suggesting high levels of gene flow at the largest scale (thousands of kilometers). However, they did find evidence of more restricted gene flow (isolation by distance) in the Eastern Caribbean. Salas et al. (2010) showed weak genetic structuring within (hundreds of kilometers) and among $(\sim 1,000 \mathrm{~km})$ regions in the Western Caribbean (Costa Rica-Panama and the Mesoamerican Barrier Reef System (MBRS), concluding that gene flow is significant within the two subregions, but likely restricted between subregions. At smaller spatial scales, Ospina-Guerrero et al. (2008) found genetic homogeneity among sites within the Columbian Caribbean $(\sim 400 \mathrm{~km})$, suggesting high levels of gene flow at this scale. However, three studies found weak (Hepburn et al. 2009; Hogan et al. 2010) to strong (Villegas-Sanchez et al. 2010) genetic structuring at a similar scale in the MBRS, showing evidence of more restricted or variable gene flow. Christie et al. (2010) found direct evidence of larval retention (i.e., self-recruitment) in Bahamian populations of $S$. partitus, on a backdrop of high gene flow among sites $(\sim 250 \mathrm{~km})$. Furthermore, two studies found that genetic structure among populations was temporally unstable at the scale of seasons (Hepburn et al. 2009) and years (Hogan et al. 2010), and this was attributed to fluctuations in connectivity among reefs (Hogan et al. 2010).

Despite recent advances, there are currently insufficient empirical data to make generalizations on expected patterns and temporal variation in connectivity among coral reefs, particularly for contemporary connectivity. Such generalizations would be valuable for validating and updating biophysical models that estimate general patterns of connectivity, and are used explicitly to design marine protected areas. Here, we empirically estimate the scale of larval dispersal, the level of self-recruitment and local retention within populations, and the spatial and temporal variation in contemporary connectivity among populations of the coral reef fish $S$. partitus at a spatial scale relevant to conservation and management. We use genetic assignment tests to track dispersal movements of individual larvae to assess connectivity among seven locations (reefs) in a $\sim 6,000 \mathrm{~km}^{2}$ region of the Mesoamerican Barrier Reef System (MBRS) in the western Caribbean (Fig. 1). We sampled from seven sites in Belize and Mexico and we repeat the sampling over three years. Here we provide the first empirical estimates of temporal variation in local 
Fig. 1 Map of the sampling sites in the Mesoamerican barrier reef system with assignment results. The map shows sampling sites from Mexico (M), Belize Barrier Reef North (BN), Turneffe Atoll North (N), Turneffe Atoll West (W), Turneffe Atoll East (E), Turneffe Atoll South (S) and Belize Barrier Reef South (BS). Pie charts indicate the proportion of assignable juveniles collected at a given site in 2005, 2006 and 2007 that were assigned to another sampled site (white), selfassigned (black), or excluded from all possible sampled populations (gray)

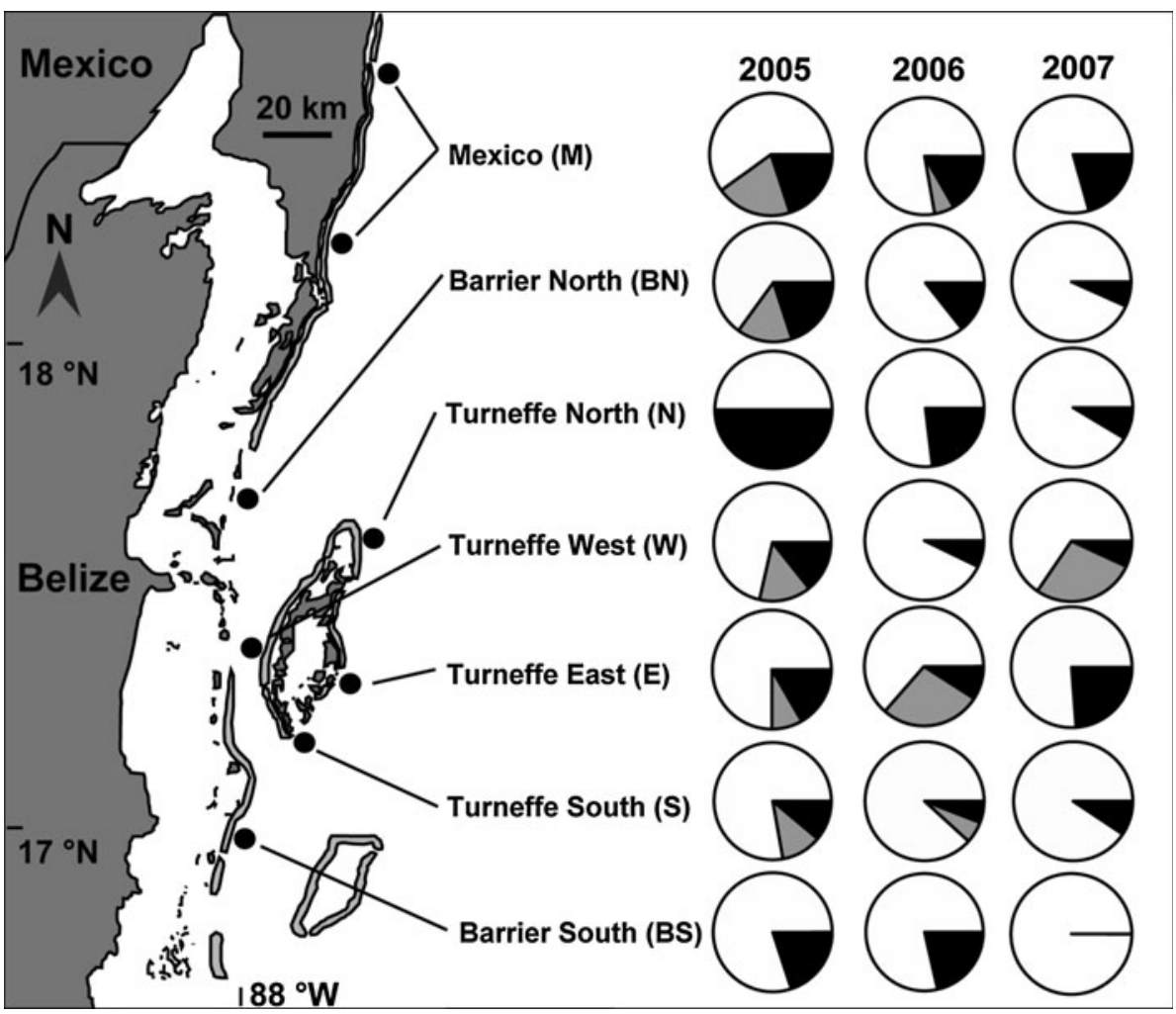

retention and connectivity among a network of marine populations.

\section{Methods}

Study species

Stegastes partitus is a small territorial damselfish (Pomacentridae) that is common on reefs throughout the tropical Western Atlantic. Adults spawn demersally following a unimodal lunar cycle with year-round reproduction and seasonal reproductive peaks from April to November (Robertson et al. 1988). The males of the species provide parental care to the eggs; however, after hatching ( $\sim 3.5$ days), larvae enter the pelagic environment and the pelagic larval duration ranges between 24 and 40 days (Robertson et al. 1988; Wellington and Victor 1989). After settlement, the adults are sedentary, defending small feeding territories indefinitely (Myrberg 1972). This life history is ideal for connectivity studies because connectivity among reefs is determined exclusively by larval dispersal.

\section{Field sampling}

We sampled seven sites in the Mesoamerican barrier reef system in the spring months each year for 3 years.
Sampling occurred from 10 June-5 July 2005, 19-29 April 2006 and 19 May-1 June 2007. At each site, approximately 100 adult $(3.8-7 \mathrm{~cm} \mathrm{TL})$ and $50-100$ recently settled juvenile (1.2-2.5 cm TL) Stegastes partitus were collected by scuba divers (Table S3). We sampled three sites along the Belize and Mexican Barrier Reef and four sites around Turneffe Atoll in Belize (Fig. 1). Due to logistical issues, the Mexican site sampled in 2005 could not be sampled in 2006, so a site was chosen further north for 2006 and 2007. All fish were collected from a site in a single day except at Turneffe East in 2005, where adults were collected over a two-week period and juveniles were collected in one day. Fin tissue was removed from each fish and preserved in RNA Later ${ }^{\circledR}$ for transport to the lab.

\section{Genotyping}

Genomic DNA was extracted from samples using a platebased extraction method (Elphinstone et al. 2003), and genotyping was performed as follows (full details are described in Hogan et al. 2010). In brief, nine microsatellite loci were chosen from the literature (Williams et al. 2003; Thiessen and Heath 2007) and screened for suitability. Polymerase chain reactions (PCR) were then performed to amplify specific loci with fluorescent dye-labeled forward primers to generate labeled PCR amplicons for sizing. The sizes of the PCR products were estimated using a LiCor 
4300 DNA analyzer with GeneImagIR 4.05 software (BD Biosciences Bioimaging).

Population genetic analyses

This study represents a novel re-analysis of previously published genotype data (Hogan et al. 2010). In this study, we focus on genetic assignment tests, and although we present basic genetic analyses here, a detailed analysis of the genetics of these populations can be found in Hogan et al. (2010). Assignment tests are used to track the dispersal of individual larvae among populations by assigning them to population-level genetic "fingerprints." Genotype data were separated into adult and juvenile populations for all three sampling seasons at each of the seven sampling sites. A fish was included in the genetic analyses if there was genotype data for at least six of the nine microsatellite loci. Exact tests for goodness of fit to Hardy-Weinberg equilibrium (HWE) using the Markov chain method (1,000 permutation burn-in followed by 100,000 permutations) for each locus within each population were performed in Arlequin v.3.11; 39 of 189 exact tests showed significant differences from HWE within adult samples after sequential Bonferroni correction (Table 1). Tests for linkage disequilibrium were performed on all populations in Genepop; approximately $2 \%$ of the tests showed significant linkage disequilibrium. Loci were tested for possible genotyping errors and molecular or biological phenomena that could explain deviations from HWE using MicroChecker v.2.2.3. Homozygote excesses were found at four of the nine loci, which explained deviations from HWE in some populations at these loci. MicroChecker results showed that homozygote excesses were not attributable to genotyping errors, but null alleles could not be ruled out;

Table 1 Summary statistics for nine microsatellite markers comparing adult populations of Stegastes partitus over three years ( $n=21$ populations)

\begin{tabular}{|c|c|c|c|c|}
\hline Locus & Global $F_{\mathrm{ST}}$ & HWE $(\%)$ & Ho & $\mathrm{Na}$ \\
\hline $\mathrm{SpGATA}_{40}$ & 0.001 & 38.0 & 0.84 & 31.1 \\
\hline $\operatorname{SpAAT}_{40}$ & 0.002 & 0.0 & 0.88 & 16.6 \\
\hline$S_{p \mathrm{AAC}_{44}}$ & 0.044 & 28.6 & 0.34 & 11.1 \\
\hline$S p \mathrm{AAC}_{33}$ & 0.002 & 28.6 & 0.77 & 17.0 \\
\hline$S p \mathrm{TG}_{16}$ & 0.006 & 28.6 & 0.87 & 29.3 \\
\hline$S p \mathrm{GGA}_{7}$ & 0.025 & 4.8 & 0.55 & 6.1 \\
\hline$S p \mathrm{TG}_{53}$ & 0.002 & 9.5 & 0.88 & 35.4 \\
\hline$S p \mathrm{TG}_{13}$ & 0.018 & 14.3 & 0.64 & 8.9 \\
\hline$S p \mathrm{GT}_{10}$ & 0.003 & 4.8 & 0.82 & 17.3 \\
\hline
\end{tabular}

HWE Percentage of adult populations that deviated from HardyWeinberg equilibrium at a particular locus (all years pooled). Ho Average heterozygosity by locus across all adult samples. Na Average number of alleles by locus across all adult samples nor could a Wahlund effect due to mixing of individuals from sources with different allele frequencies. We do not account for null alleles in our analyses because it is unlikely that null alleles are driving these homozygote deficits. In this study, no locus deviated from HWE in more than 8 of 21 adult populations, and deviations from HWE were not consistent across years-evidence that null alleles are an unlikely cause of disequilibrium. A Wahlund effect is much more likely to be causing HW disequilibrium; in fact, a detailed study of our populations found strong evidence of a Wahlund effect driving deviations from HWE (Hogan et al. 2010). Furthermore, a previous study of S. partitus (Purcell et al. 2009) suggested homozygote excesses in their populations were likely attributable to a Wahlund effect, but they ruled it out only because genetic differentiation among sites was low. However, it has been shown that even weakly divergent populations can experience a Wahlund effect (Johnson and Black 1984; Selkoe et al. 2006; Hogan et al. 2010). Regardless, Carlsson (2008) has shown that null alleles have only a weak effect on genetic assignment tests, and they result in a loss of assignment power. Our method of exclusion (see below) is robust to this effect by excluding individuals that have poor assignment power. Finally, $F$-statistics were calculated to quantify the extent of genetic heterogeneity among all populations within each year using MSA 4.05. Global $F_{\text {ST }}$ values were low for each year (2005: 0.007, 2006: 0.001, 2007: 0.009), but were significantly different than zero in 2005 and 2007. Pair-wise (among sampling sites, within year) $F_{\mathrm{ST}}$ values ranged between 0.001 and 0.020 (Table $\mathrm{S} 4)$.

Genotype assignment method

Juveniles were assigned to putative adult populations based on their multi-locus genotype probabilities using a two-step method. First, a Bayesian assignment method was used (Rannala and Mountain 1997), with probability estimates generated by 10,000 iterations of Monte Carlo re-sampling using a published algorithm (Paetkau et al. 2004) in Geneclass v.2.0. Second, a rank-based assignment method was applied where individuals are assigned based on their negative log-likelihood of belonging to one population relative to the other source populations (Rannala and Mountain 1997). Individuals were considered to be assigned when they met two criteria:

1. They must be assigned to at least one source population with a probability of $>85 \%$ in the first Monte Carlo based assignment procedure (threshold determined in a sensitivity analysis described below). Individuals with a $<5 \%$ probability of belonging to any site were excluded from belonging to any of the 
source populations, and those between $5 \%$ and the threshold value $(85 \%)$ were considered unassigned.

2. The individuals that met the $85 \%$ threshold in the first step were then assessed using the rank-based method. Individuals were assigned to the source population if the likelihood of assignment to the highest ranked source population was $50 \%$ greater than the likelihood of assignment to the next highest ranked population.

Ten independent iterations of the assignment tests were conducted, and only individuals that were assigned to the same site in a majority of these iterations were considered successfully assigned individuals. This procedure further increased the stringency of assignment and eliminated individuals assigned to more than one potential source site. Our analysis results in all juvenile fish being classified as (a) successfully assigned, (b) successfully excluded from all sites, or (c) unassignable with any confidence. The removal from further analysis of juveniles that failed to assign should not create a biologically meaningful bias in our dispersal and connectivity data. A biological bias could arise if there are certain genotypes that are prone to different dispersal modes (i.e., greater or lesser dispersal distance) and if certain genotypes are preferentially removed by the analysis. However, in a previous study, we found that our genetic markers are neutral to selection in the populations studied here (Hogan et al. 2010), and it is unlikely that biologically meaningful biases exist among our assigned juveniles (relative to the sampled population). Furthermore, the sample size of assignable juveniles ( $n=453$ ) remains large, and thus we expect that our dispersal estimates are robust and representative of the sampled populations.

\section{Assignment sensitivity analysis}

We used an assignment sensitivity analysis to objectively determine the threshold of assignment stringency that we used in the first step of our assignment analysis. Because of the low-level genetic differentiation among our sites and across the entire Western Caribbean (Purcell et al. 2009), there are likely to be errors in the assignment of some juveniles, especially those with common genotypes. We used an information criterion to set an assignment threshold to reduce the number of erroneous juvenile assignments and at the same time to maximize sample size to increase statistical power. We assigned only those juveniles that met the determined threshold of assignment stringency, those that have high levels of certainty in their assignment. First we ran the assignment tests (as described above) using adult and juvenile samples from 2007 at ten different levels of assignment stringency (thresholds ranging from 5 to 95\%). Next we plotted the percentage of juveniles assigned at each threshold (Fig. S1). We found a trend toward a general decline in the percent of juveniles assigned as assignment stringency increases (Fig. S1). Next we plotted the rate of change in the percent of juveniles assigned between successive thresholds (Fig. S2). This clearly shows where the greatest difference in percent assignment between successive thresholds occurs. The greatest difference in the percent of juveniles included in the assignment analysis occurred between the $95 \%$ threshold and the $85 \%$ threshold. This difference is more than double that of any other difference between successive thresholds. Therefore, the $85 \%$ assignment threshold provides the greatest balance between stringency of assignment and sample size.

\section{Dispersal distance analysis}

We calculated expected, random distributions of dispersal distance (assuming straight-line paths between pairs of sampling sites) to test whether the observed distributions were different from those expected due to random dispersal. Because the distances between sampling sites were not evenly distributed among distance categories (bins), we calculated the expected frequencies of dispersal events based on the proportion of possible dispersal pathways between sampled sites in each distance bin. We compared observed dispersal distance distributions to the expected distribution using Kolmogorov-Smirnov tests (Fig. 2b).

\section{Dispersal direction analysis}

We calculated the net dispersal direction vector in each year to test for directionality in dispersal. Each individual dispersal event was assigned to a direction bin (in $22.5^{\circ}$ increments). Because the directions between sampling sites were not evenly distributed among direction bins, we calculated the expected frequencies of dispersal events based on the proportion of possible dispersal pathways between sampled sites in each direction bin (assuming straight-line dispersal). Directional frequencies were corrected in the same manner as for distance (above), and net dispersal direction was then calculated as the sum of all corrected dispersal directions (red arrows; Fig. 2c).

\section{Analysis of randomized adult populations}

Using the 2007 data, we created 10 randomized adult populations in order to test the validity of the assignments made by the software program Geneclass 2.0. Adult multilocus genotypes were preserved, but they were assigned randomly among our seven sampling sites, preserving the original sample sizes. The juvenile genotypes were then assigned to the randomized genotype "populations" 

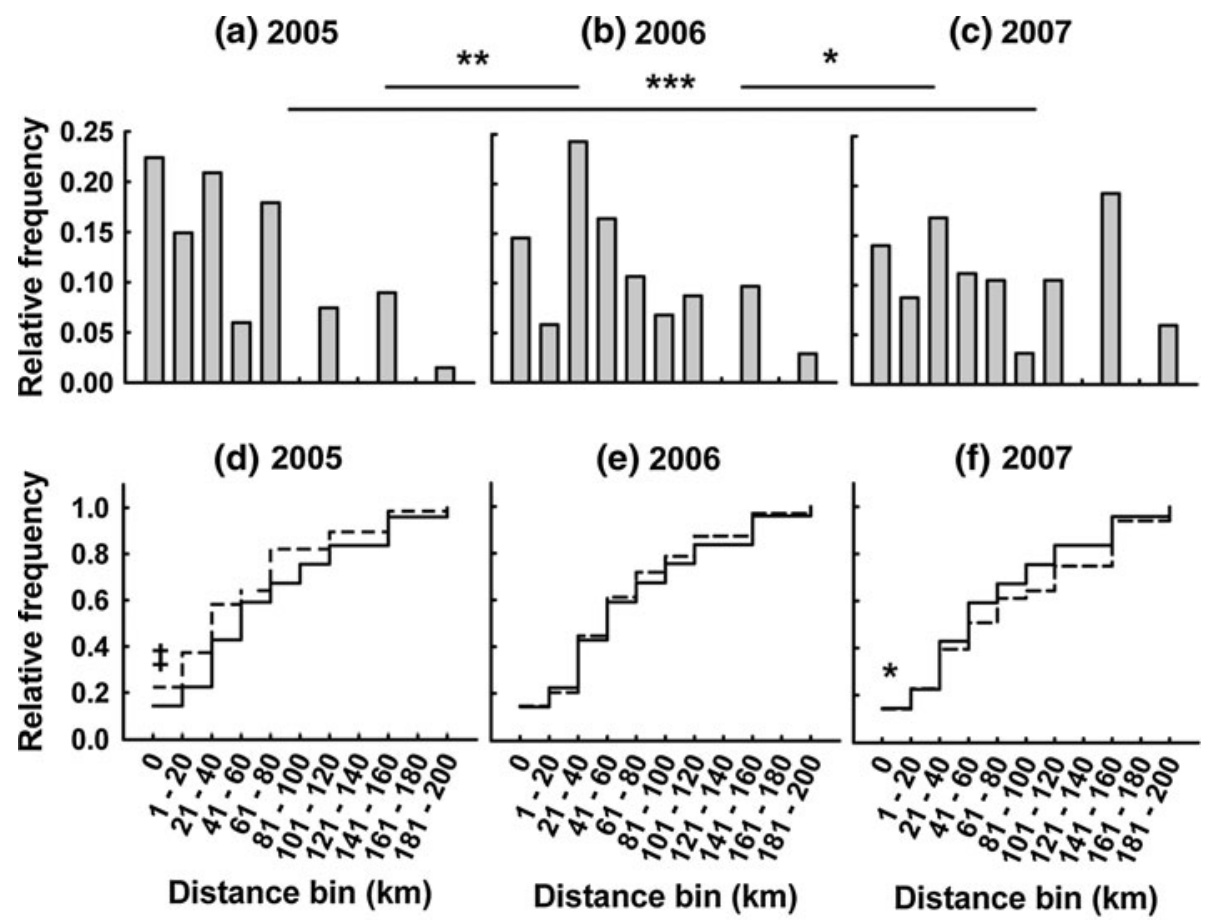

(f) 2007

(g) 2005
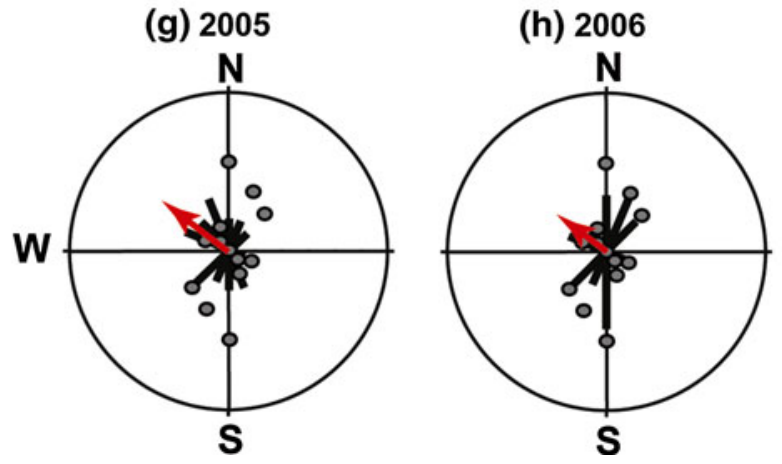

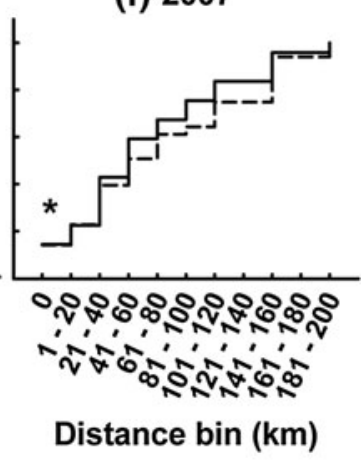

(i) 2007

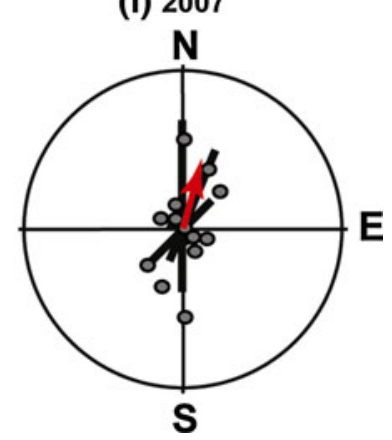

Fig. 2 Spatial and temporal dispersal within the Mesoamerican Barrier Reef System. All data for a given year are presented in the same column. a-c Frequency distributions of dispersal distances. Significant differences among years in distribution were determined by Kolmogorov-Smirnov tests $(k=11, n=99)$ and are shown as bars above the graphs $\left(* P<0.05, * * P<0.01\right.$, $\left.{ }^{* * *} P<0.001\right)$. d-f Cumulative frequency distributions of expected, random dispersal (solid line) and observed dispersal (dashed line) with increasing dispersal distance. Significant differences between observed and

following the assignment method described above. We compared the dispersal kernels generated from these ten randomizations with the results yielded by the actual data. To test for a statistical difference between random assignment and actual assignment results, we compared chi-squared distributions between random kernels and the actual data. The chi-squared distribution for the ten random kernels was generated by comparing each kernel to all other random kernels and generating chi-squared values for each comparison. Next we compared the actual dispersal random distributions were determined by Kolmogorov-Smirnov tests $\left(k_{\mathrm{all}}=11, n_{2005}=67 ; n_{2006}=103 ; n_{2007}=286\right)$ and are indicated on the figure $(* P<0.05, *$ power analysis indicates significant difference after small $8 \%$ increase in sample size). g-i Frequency distributions of dispersal directions (assuming straight line dispersal). Gray dots indicate the null expected frequencies based on the distribution of our sampling sites. Red arrows indicate the net direction of dispersal after correction for biases in dispersal among directional categories

kernel to each of the ten random kernels, generating a chisquared value for each comparison. We then compared the two chi-squared distributions to each other to test for a difference between random and actual assignment results. Both chi-squared distributions were approximately normal (i.e., skew and kurtosis $-1>x<1$ ), and the two distributions did not overlap at their $95 \%$ confidence intervals, so the actual assignment results were significantly different from results generated by assignment to randomly generated adult populations. 


\section{Results}

Neutral microsatellite DNA markers were used to characterize adult (source) populations, and genotype assignment analyses were used to link individual juveniles back to their likely natal reefs. We genotyped 1,828 adult fishes from seven reefs, and re-sampled every year for three years. The average pair-wise $F_{\mathrm{ST}}$ value among adult populations was 0.005 , and the maximum pair-wise $F_{\mathrm{ST}}$ value was 0.02 over the three years of sampling. Of the 1,291 juvenile samples collected and genotyped over the three years of sampling, $456(35 \%)$ were assigned to a particular sampled site with high confidence. Of the successfully assigned juveniles, 70 $(15 \%)$ were assigned back to the site at which they were caught (i.e., "returned home"), and the remaining 386 (85\%) juveniles were assigned to one of the other sampled sites. The degree of self-recruitment (the ratio of juveniles returning home to juveniles coming from other source reefs) varied widely both among sites and across time (Fig. 1); however, the average self-recruitment rate across the region over all years was $15 \%$, and was relatively consistent among the three years (2005: 22\%; 2006: 15\%; 2007: 14\%; Fig. 2a). By comparison, the degree of local retention (the ratio of juveniles produced at a site that returned home to the total number of juveniles produced at that site) was on average higher than self-recruitment $(21 \%)$, but yearly estimates were similar to estimates of self-recruitment (2005: 35\%; 2006: 16\%; 2007: 13\%), albeit with notably higher local retention in 2005. When we enlarged our spatial scale from the site level, we found that an average of $65 \%$ of all assignable juveniles produced on Turneffe Atoll recruited back to populations on Turneffe over the three years of sampling, but local retention at this scale varied widely among years (2005: 84\%; 2006: 70\%; 2007: 58\%). We also found that $1.7 \%(n=22)$ of all juveniles sampled were excluded from all sampled sites with high confidence.

The distribution of the calculated dispersal distances (dispersal kernels) differed significantly among sampling years (Fig. 2), while the relative contribution of each source population to the recruitment at a given site changed dramatically over time (Fig. 3a). If there were predictable patterns of connectivity from year to year, we would expect to see a positive relationship in the strength of interaction between site pairs from one year to the next. However, we found that there was no predictability in the larval dispersal pathways from one year to the next (Fig. 3b). Also, 18\% of the variation in self-recruitment was partitioned among years, and $26 \%$ was explained by the site-by-year interaction (log-linear analysis: $\Delta$ chi-squared: $d f_{\text {years }}=2, \Delta \chi^{2}=99.9$, $\left.p<0.0001 ; d f_{\text {site } \times \text { year }}=12, \Delta \chi^{2}=22.3, p=0.051\right)$.

As a species with a larval duration of approximately 30 days, Stegastes partitus has the potential for long-distance dispersal. In our system, dispersing fish, sampled over the three years, traveled an average of $77 \mathrm{~km}$ $(\mathrm{SEM} \pm 6 \mathrm{~km})$. The majority of assignable juveniles recruited within a radius of $60 \mathrm{~km}$ or less around the natal reef in each year (Fig. 2a). Five percent $(n=21)$ of assignable larvae were shown to have traveled the full extent of our sampling region $(187 \mathrm{~km})$ during their larval lives. Furthermore, there was generally no decline in the strength of connectivity among sites with distance from the source (Fig. 3c).

We used the distributions of dispersal distances (dispersal kernels) and dispersal direction to test for patterns within and across the sample years. We test these patterns against null model expectations. The mean dispersal kernel in 2007 was significantly different from random expectation (Fig. 2b), and a power analysis revealed that, with only a small (8\%) increase in sample size, the 2005 distribution was also significant (Fig. 2b). Deviation from random expectation in 2005 was driven by greater than expected short-distance recruitment, and in 2007 differences were driven by lower than expected levels of recruitment to middle distances (Fig. 2b). The 2006 distribution was not significantly different from random. When our results were compared to those of assignments to randomized adult populations, the observed dispersal kernels differed significantly from those generated from randomized trials (see "Methods"). Furthermore, the direction of dispersal was also not random, but rather biased in a northwesterly direction in both 2005 and 2006 and in a north-northeasterly direction in 2007 (Fig. 2c). In 2006 the overall directionality of dispersal was weak.

\section{Discussion}

Marine protected areas typically have two primary objectives: to conserve biodiversity and to enhance fishery yields. For both ends, quantified patterns of connectivity, estimates of self-recruitment and local retention, and measures of dispersal distance are important for determining the size and spacing of reserves that will maximize their effectiveness (Fogarty and Botsford 2007; Jones et al. 2007). Here we used highly polymorphic microsatellite markers and genetic assignment tests to identify natal sources of recently dispersed larvae of the coral reef fish Stegastes partitus.

We were able to identify or exclude natal sources for approximately $35 \%$ of all fishes sampled with a high degree of certainty. Our results show that self-recruitment occurs consistently at appreciable levels among natural populations of a common reef fish, the bicolor damselfish. Our data support the view that self-recruitment is a common and regular phenomenon in natural coral reef fish populations (Jones et al. 2005; Almany et al. 2007; Christie 
(a) 2005

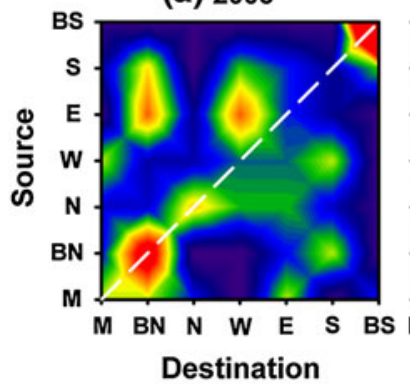

(d) 2005 vs. 2006

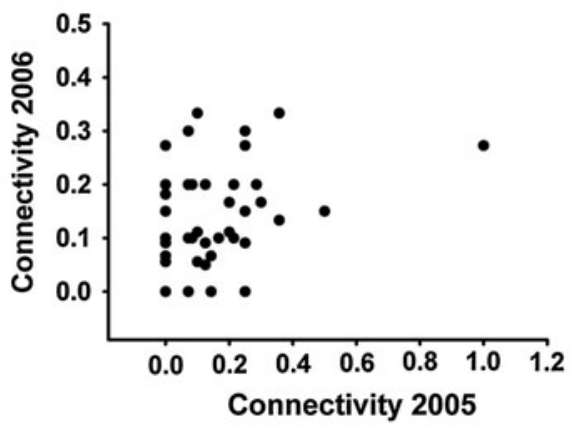

(b) 2006

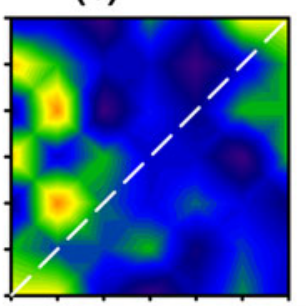

Destination (c) 2007

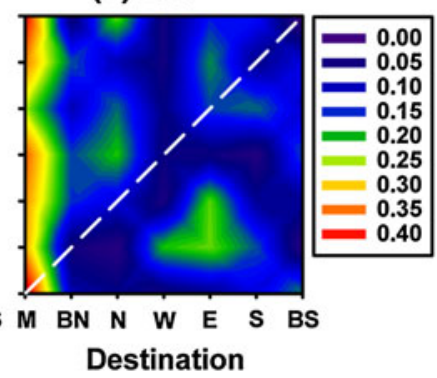

(e) 2006 vs. 2007

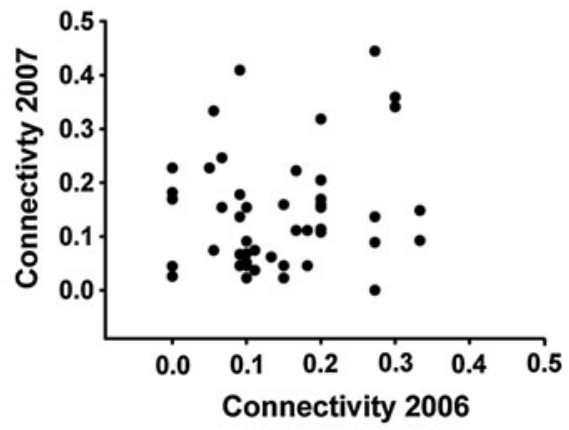

(f) 2005

(g) 2006

(h) 2007

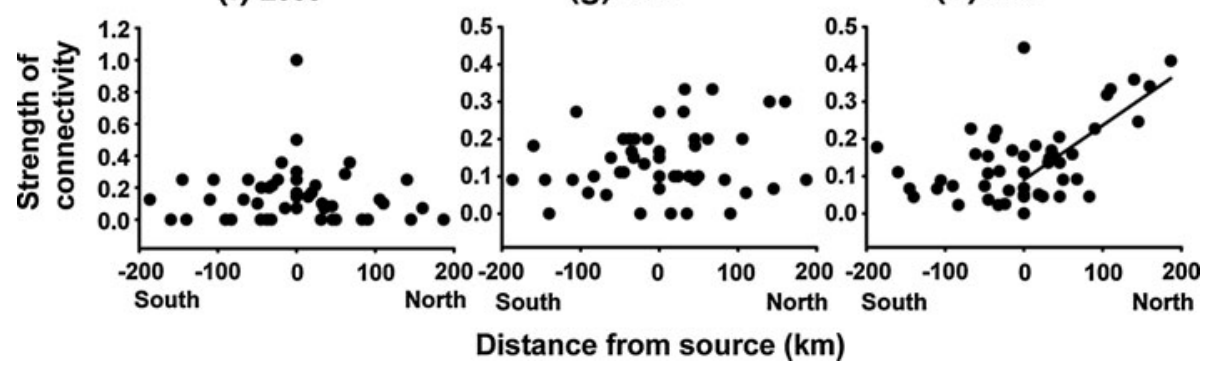

Fig. 3 Spatial and temporal connectivity and source-destination interactions. a-c Matrices of source-destination dynamics; contours indicate proportions of juveniles collected at a destination site (columns) that were assigned to a particular source site (rows). The proportions in each row add to give 1 . The diagonal dashed line indicates local retention at a particular site. Axis site codes are the same as the codes in Fig. 1. d, e Change over time in sourcedestination interactions from one study year to the next. Plotted values are the strength of the source-destination connectivity measured as the proportion of juveniles produced at a source site (in a given year) that were collected at a particular destination population. If there was temporal consistency in connectivity we

et al. 2010). Previous studies have estimated that selfrecruitment to populations of coral reef fishes ranges from 15 to $60 \%$ for a single reef (Jones et al. 1999, 2005; Swearer et al. 1999; Almany et al. 2007). Our estimates range from 0 to $50 \%$ at individual reefs, and the average level of self-recruitment is $15 \%$. However, spatial and temporal variability at the individual reef level underscores the unpredictability of self-recruitment at small scales. Therefore, spatially and temporally replicated estimates of self-recruitment are necessary to capture this variability. would expect to see a positive relationship between the sourcedestination interactions in consecutive years. $\mathbf{f}-\mathbf{h}$ Variation in the strength of connectivity with distance from the source population (origin). The strength of connectivity is measured as the proportion of individuals produced at a particular source population. Positive distances from the origin are in a northerly direction and negative distances are in a southerly direction. The null expectation is an isolation-by-distance model whereby the strength of connectivity among sites declines with increasing distance. The solid line (h) indicates a relationship with a slope that is significantly different from zero (2007 North: $r^{2}=0.41, F=18.2, P<0.01$ ). There were no other significant relationships with geographic distance (f, g)

By comparison, the levels of local retention at the site level were similar to levels of self-recruitment, albeit higher on average in 2005. Local retention is an estimate of the demographic independence of populations. If local retention is high, then the population is less reliant on larval subsidies from other populations, while selfrecruitment is not explicitly an estimate of demographic independence. Therefore, it is interesting that our estimates of self-recruitment and local retention are similar, because this suggests that self-recruitment might be a good 
estimator of local retention. As we scale up from the site level, we find that $65 \%$ of larvae produced at sites within Turneffe Atoll returned to populations in Turneffe. While the level of local retention reported here is not high enough to drive isolation (and genetic divergence) at the site scales, our results show that local retention plays a significant role in the replenishment of these populations, and that demographic independence increases with spatial scale. However, our estimates of local retention varied among years at both the site and atoll scales, suggesting that the influence of recruitment subsidies from neighboring populations and the level of demographic independence of populations vary temporally.

We found that the average dispersing larva traveled $\sim 77 \mathrm{~km}$ from the natal reef within this study region. However, the strength of connectivity between site pairs did not decline with distance, suggesting that larvae may disperse further than the boundaries of our sampling area. The $77 \mathrm{~km}$ distance found here is a conservative estimate of the average dispersal distance of larvae. In fact, a small percentage of the juveniles sampled (1.7\%) were excluded from all possible sampling sites, suggesting that these fish were perhaps spawned in unsampled populations with markedly different genetic structure than any of our sampled populations, perhaps dispersing from some distant location. Long-distance dispersers may have a significant impact on genetic structure, acting to homogenize population structure over large geographic areas (Wright 1931). This is the case for Stegastes partitus, which shows weak genetic differentiation across the entire Caribbean basin (Purcell et al. 2009).

Our results suggest that significant demographic connectivity can occur at a spatial scale of $\sim 200 \mathrm{~km}$, and we found no evidence of a decline in the strength of connectivity with distance from the source population. Therefore, populations within the boundaries of a marine reserve can, perhaps, provide recruitment subsidies to areas as distant as $\sim 200 \mathrm{~km}$ or greater from the reserve. Generally, the spatial scale of demographic connectivity in this system appears to match predictions from hydrodynamic models (Cowen et al. 2006) and recent empirical data (Becker et al. 2007; Planes et al. 2009) which suggest that populations are likely to receive significant larval inputs primarily from sources spaced less than $100 \mathrm{~km}$ away. The observed dispersal directions also appear to match model predictions. In 2005 and 2006, dispersal directions reflected the average prevailing currents in this region during the spring months (Tang et al. 2006; Soto et al. 2009). The variation from this pattern in 2007 likely reflects variability in regional current patterns not captured by models that average current patterns.

It has been shown in studies of natural and simulated populations that genetic assignment tests can be highly accurate in identifying source populations (Berry et al. 2004; Waples and Gaggiotti 2006). Assignment accuracy is related to the migration rate, and as a correlate, the genetic differentiation between subpopulations. The higher the migration rate between subpopulations, the more genetically similar those populations become, and the less accurate assignment will be (Berry et al. 2004; Waples and Gaggiotti 2006). However, studies have shown that even at quite low levels of differentiation $\left(F_{\mathrm{ST}} \approx 0.01-0.02\right)$, similar to those found among some of our populations, assignment tests can be quite accurate (Berry et al. 2004; Waples and Gaggiotti 2006). Furthermore, using many highly polymorphic loci and applying stringent threshold criteria to the assignment tests (as we have done here) reduces the percentage of incorrect assignments and improves assignment accuracy (Bjornstad and Roed 2002; Berry et al. 2004; Waples and Gaggiotti 2006). Also, the accuracy of assignments could be reduced if the allele frequency distributions of unsampled populations are very similar to one or more of our sampled populations, causing larvae to be falsely assigned to a site they did not actually come from. It is not logistically possible to sample all potential source populations for a marine fish such as $S$. partitus, which is very abundant and broadly distributed throughout the Caribbean. However, recent studies have shown that it is very unlikely that unsampled populations would have the same allele frequency distribution as one of our sites. A recent study of our populations showed that there were significant differences in allele frequency distributions among all of our sites, and weak but significant levels of genetic differentiation (Hogan et al. 2010). Other studies support this, showing weak to strong genetic differentiation among populations of $S$. partitus in the MRBS region (Hepburn et al. 2009; Salas et al. 2010; VillegasSanchez et al. 2010). Furthermore, our exclusion-assignment procedure is highly stringent and excludes individuals that have very common genotypes (those more likely to cause a false assignment). Finally, if our assignments were erroneous due to low assignment power, the patterns of the assignments would be random (Waples and Gaggiotti 2006), but we show that our patterns are significantly different from that expected at random. Overall, we cannot measure the effect of unsampled sites on assignment accuracy, and we acknowledge that some assignment errors are likely. However, we argue that this assignment procedure is highly stringent and robust, and that the overall patterns found are real.

The technique used here can be very useful for obtaining temporally replicated empirical estimates of connectivity for many intractable species-a task that will be necessary to validate predictive models of connectivity and ultimately provide general connectivity information for better marine management practices. We found that dispersal distance 
distributions, dispersal directions, and the patterns of connectivity among sites were not stable through time. The unstable temporal pattern of connectivity indicates that consistent source-sink patterns do not exist for this species in this region. Given the nature of the temporal variability in connectivity, our results suggest that models estimating connectivity should match directly with the time frames of empirical studies to get the best validation of these models.

Temporal variability in connectivity has implications for the management and conservation of marine species. Variable connectivity can increase instability of population genetic structure (Hedgecock 1994; Larson and Julian 1999; Selkoe et al. 2006; Hogan et al. 2010). Furthermore, variability in source-sink interactions can act to stabilize metapopulations and prevent the extinction of local populations (Holland and Hastings 2008). Our results highlight the need for temporal connectivity data in other marine species, and emphasize the need for modeling studies to explore the effects of temporal variability in dispersal (Botsford et al. 2009). If temporal variability in the pattern and extent of connectivity is found to be a common phenomenon among reef fish populations, connectivity data from several years would be necessary for confidence in any source-sink patterns, especially when using this information explicitly for marine reserve network design.

Acknowledgments This work was supported by the Global Environment Facility-funded University of Queensland and World Bank project Coral Reef Targeted Research for Capacity Building and Management, by a Natural Sciences and Engineering Research Council Tier I Canada Research Chair grant to D.D.H., as well as a GLIER postdoctoral fellowship from the University of Windsor to J.D.H. We thank the University of Belize, Institute of Marine Studies, and D. Campbell-Smith for logistical support during the field collections; P. Usseglio, E. Salas, D. Hasselman and R. Walter for help with field collections; S. Jaimeson and R. Walter for statistical help; R. Walter for discussion; and S. Jamieson, S. Ellis, R. Walter, T. Pitcher, S. Planes and anonymous reviewers for comments on the manuscript.

\section{References}

Almany GR, Berumen ML, Thorrold SR, Planes S, Jones GP (2007) Local replenishment of coral reef fish populations in a marine reserve. Science 316:742-744. doi:10.1126/science.1140597

Becker BJ, Levin LA, Fodrie FJ, McMillan PA (2007) Complex larval connectivity patterns among marine invertebrate populations. Proc Natl Acad Sci USA 104:3267-3272. doi:10.1073/ pnas.0611651104

Berry O, Tocher MD, Sarre SD (2004) Can assignment tests measure dispersal? Mol Ecol 13:551-561. doi:10.1046/j.1365-294X. 2004.02081.x

Bjornstad G, Roed KH (2002) Evaluation of factors affecting individual assignment precision using microsatellite data from horse breeds and simulated breed crosses. Anim Gen 33:264-270. doi:10.1046/j.1365-2052.2002.00868.x
Botsford LW et al (2009) Connectivity and resilience of coral reef metapopulations in marine protected areas: matching empirical efforts to predictive needs. Coral Reefs 28:327-337. doi: 10.1007/s00338-009-0466-z

Bradbury IR, Laurel B, Snelgrove PVR, Bentzen P, Campana SE (2008) Global patterns in marine dispersal estimates: the influence of geography, taxonomic category and life history. Proc R Soc Lond B 275:1803-1809. doi:10.1098/rspb.2008.0216

Carlsson J (2008) Effects of null alleles on assignment testing. J Hered 99:616-623. doi:10.1093/jhered/esn048

Christie MR, Johnson DW, Stallings CD, Hixon MA (2010) Selfrecruitment and sweepstakes reproduction amid extensive gene flow in a coral-reef fish. Mol Ecol 19:1042-1057. doi:10.1111/j. 1365-294X.2010.04524.X

Cowen RK, Paris CB, Srinivasan A (2006) Scaling of connectivity in marine populations. Science 311:522-527. doi:10.1126/science. 1122039

Eckert GL (2003) Effects of the planktonic period on marine population fluctuations. Ecology 84:372-383. doi:10.1890/00129658(2003)084[0372:EOTPPO]2.0.CO;2

Elphinstone MS, Hinten GN, Anderson MJ, Nock CJ (2003) An inexpensive and high-throughput procedure to extract and purify total genomic DNA for population studies. Mol Ecol Notes 3:317-320. doi:10.1046/j.1471-8286.2003.00397.x

Fisher R, Bellwood DR (2003) Undisturbed swimming behaviour and nocturnal activity of coral reef fish larvae. Mar Ecol Prog Ser 263:177-188. doi:10.3354/meps263177

Fogarty MJ, Botsford LW (2007) Population connectivity and spatial management of marine fishes. Oceanogr 20:112-123

Hedgecock D (1994) Temporal and spatial genetic structure of marine animal populations in the California Current. Calif Coop Ocean Fish Inv 35:73-81

Hepburn RI, Sale PF, Dixon B, Heath DD (2009) Genetic structure of juvenile cohorts of bicolor damselfish (Stegastes partitus) along the Mesoamerican barrier reef: chaos through time. Coral Reefs 28:277-288. doi:10.1007/s00338-008-0423-2

Hogan JD, Thiessen RJ, Heath DD (2010) Variability in connectivity indicated by chaotic genetic patchiness within and among populations of a marine fish. Mar Ecol Prog Ser 417:263-275. doi: $10.3354 /$ meps08793

Holland MD, Hastings A (2008) Strong effect of dispersal network structure on ecological dynamics. Nature 456:792-794. doi: 10.1038/nature07395

Hutchings JA (2000) Collapse and recovery of marine fishes. Nature 406:882-885. doi:10.1038/35022565

Jackson JBC et al (2001) Historical overfishing and the recent collapse for coastal ecosystems. Science 293:629-638. doi: 10.1126/science. 1059199

Johnson MS, Black R (1984) The Wahlund effect and the geographical scale of variation in the intertidal limpet Siphonaria sp. Mar Biol 79:295-302

Jones GP, Milicich MJ, Emslie MJ, Lunow C (1999) Self-recruitment in a coral reef fish population. Nature 402:802-804. doi: $10.1038 / 45538$

Jones GP, Planes S, Thorrold SR (2005) Coral reef fish larvae settle close to home. Curr Biol 15:1314-1318. doi:10.1016/j.cub. 2005.06.061

Jones GP, Srinivasan M, Almany GR (2007) Population connectivity and conservation of marine biodiversity. Oceanogr 20:100-111

Jones GP et al (2009) Larval retention and connectivity among populations of corals and reef fishes: history, advances and challenges. Coral Reefs 28:307-325. doi:10.1007/s00338-0090469-9

Larson RJ, Julian RM (1999) Spatial and temporal genetic patchiness in marine populations and their implications for fisheries management. Cal Coop Ocean Fish 40:94-99 
Leis JM (1991) The pelagic stage of reef fishes: the larval biology of coral reef fishes. In: Sale PF (ed) The ecology of fishes on coral reefs. Academic, San Diego, pp 183-230

Levin LA (2006) Recent progress in understanding larval dispersal: new directions and digressions. Integr Comp Biol 46:282-297. doi:10.1093/icb/024

Miller JA, Shanks AL (2004) Evidence for limited larval dispersal in black rockfish (Sebastes melanops): implications for population structure and marine reserve design. Can J Fish Aquat Sci 61:1723-1735. doi:10.1139/f04-111

Myrberg AA (1972) Ethology of the bicolor damselfish, Eupomacentrus partitus: a comparative analysis of laboratory and field behaviour. Anim Behav Monogr 5:197-283

Ospina-Guerrero SP, Landinez-Garcia RM, Rodriguez-Castro DJ, Arango R, Marquez E (2008) Genetic connectivity of Stegastes partitus in the south Caribbean evidenced by microsatellite analysis. Cien Mar 34:155-163

Paetkau D, Slade R, Burden M, Estoup A (2004) Genetic assignment methods for the direct, real-time estimation of migration rate: a simulation-based exploration of accuracy and power. Mol Ecol 13:55-65. doi:10.1046/j.1365-294X.2003.02008.x

Planes S, Jones GP, Thorrold SR (2009) Larval dispersal connects fish populations in a network of marine protected areas. Proc Natl Acad Sci USA 106:5693-5697. doi:10.1073/pnas.0808007106

Purcell JFH, Cowen RK, Hughes CR, Williams DA (2009) Population structure in a common Caribbean coral-reef fish: implications for larval dispersal and early life history traits. J Fish Biol 74:403-417. doi:10.1111/j.1095-8649.2008.02078.x

Rannala B, Mountain JL (1997) Detecting immigration by using multilocus genotypes. Proc Natl Acad Sci USA 94:9197-9201

Robertson DR, Green DG, Victor BC (1988) Temporal coupling of production and recruitment of larvae of a Caribbean reef fish. Ecology 69:370-381

Salas E, Molina-Urena H, Walter RP, Heath DD (2010) Local and regional genetic connectivity in a Caribbean coral reef fish. Mar Biol 157:437-445. doi:10.1007/s00227-009-1330-y

Sale PF (1980) The ecology of fishes on coral reefs. Oceanogr Mar Biol Ann Rev 18:367-421

Sale PF et al (2005) Critical science gaps impede use of no-take fishery reserves. Trends Ecol Evol 20:74-80. doi:10.1016/j.tree. 2004.11.007

Selkoe KA, Gaines SD, Caselle JE, Warner RR (2006) Current shifts and kin aggregation explain genetic patchiness in fish recruits. Ecology 87:3082-3094. doi:10.1890/0012-9658(2006)87[3082: CSAKAE]2.0.CO;2
Shima JS, Swearer SE (2009) Larval quality is shaped by matrix effects: implications for connectivity in a marine metapopulation. Ecology 90:1255-1267. doi:10.1890/08-0029.1

Soto I et al (2009) Physical connectivity in the Mesoamerican barrier reef system inferred from 9 years of ocean color observations. Coral Reefs 28:415-425. doi:10.1007/s00338-009-0465-0

Swearer SE, Caselle JE, Lea DW, Warner RR (1999) Larval retention and recruitment in an island population of a coral reef fish. Nature 402:799-802. doi:10.1038/45533

Tang LQ, Sheng JY, Hatcher BG, Sale PF (2006) Numerical study of circulation, dispersion, and hydrodynamic connectivity of surface waters on the Belize shelf. J Geophys Res Oceans 111. doi: 10.1029/2005JC002930

Thiessen RJ, Heath DD (2007) Characterization of one trinucleotide and six dinucleotide microsatellite markers in bicolor damselfish, Stegastes partitus, a common coral reef fish. Conserv Genet 8:983-985. doi:10.1007/s10592-006-9207-9

Thorrold SR et al (2002) Quantifying larval retention and connectivity in marine populations with artificial and natural markers. Bull Mar Sci 70:291-308

Villegas-Sanchez CA, Rivera-Madrid R, Arias-Gonzalez JE (2010) Small scale genetic connectivity of bicolor damselfish (Stegastes partitus) recruits in Mexican Caribbean reefs. Coral Reefs 29:1023-1033. doi:10.1007/s00338-010-0643-0

Walter RP, Aykanat T, Kelly DW, Shrimpton JM, Heath DD (2009) Gene flow increases temporal stability of Chinook salmon (Oncorhynchus tshawytscha) populations in the Upper Fraser River, British Columbia, Canada. Can J Fish Aquat Sci 66:167-176. doi:10.1139/F08-201

Waples RS, Gaggiotti O (2006) What is a population? An empirical evaluation of some genetic methods for identifying the number of gene pools and their degree of connectivity. Mol Ecol 15:1419-1439. doi:10.1111/j.1365-294X.2006.020890.x

Wellington GM, Victor BC (1989) Planktonic larval duration of one hundred species of Pacific and Atlantic damselfishes (Pomacentridae). Mar Biol 101:557-567

Williams DA, Purcell J, Hughes CR, Cowen RK (2003) Polymorphic microsatellite loci for population studies of the bicolor damselfish, Stegastes partitus (Pomacentridae). Mol Ecol Notes 3:547-549. doi:10.1046/j.1471-8286.2003.00506.x

Wright S (1931) Evolution in Mendelian populations. Genetics 16: 97-158 